\title{
Non-leptonic B-decays at two loops in QCD Factorization
}

\section{Guido Bell}

Naturwissenschaftlich-Technische Fakultät, Universität Siegen,

Walter-Flex-Str. 3, 57068 Siegen, Germany

E-mail: bellaphysik.uni-siegen.de

\section{Martin Beneke}

Physik Department T31, James-Franck-Straße 1,

Technische Universität München,

D-85748 Garching, Germany

E-mail: mbeneke@tum.de

\section{Tobias Huber*}

Naturwissenschaftlich-Technische Fakultät, Universität Siegen,

Walter-Flex-Str. 3, 57068 Siegen, Germany

E-mail: huber@physik.uni-siegen.de

\section{Xin-Qiang Li}

Institute of Particle Physics and Key Laboratory of Quark and Lepton Physics (MOE),

Central China Normal University,

Wuhan, Hubei 430079, P. R. China

E-mail: xqli@mail.ccnu.edu.cn

We report on the calculation of the two-loop penguin amplitudes in non-leptonic B decays in the framework of QCD factorization. We discuss the computation of this genuine two-loop, twoscale problem and provide details on the matching from QCD onto SCET, the evaluation of the master integrals, and the convolution of the hard scattering kernels with the light-cone distribution amplitude of the light meson. Preliminary results on the size of the two-loop correction are given.

14th International Symposium on Radiative Corrections (RADCOR2019)

9-13 September 2019

Palais des Papes, Avignon, France

\footnotetext{
*Speaker.
} 


\section{Introduction}

Charmless non-leptonic decays of $B_{(s)}$ mesons play an important role in quantifying the amount of $\mathrm{CP}$ violation, one of the most subtle phenomena in flavour physics. The structure of the decay amplitude can be generically written as

$$
\mathscr{A}(\bar{B} \rightarrow f)=\lambda_{u}^{(D)} A_{f}^{u}+\lambda_{c}^{(D)} A_{f}^{c}=\sum_{i}\left[\lambda_{\mathrm{CKM}} \times C \times\langle f|\mathscr{O}| \bar{B}\rangle_{\mathrm{QCD}+\mathrm{QED}}\right]_{i} .
$$

The main elements of this formula are i) the Wilson coefficients $C$ of tree or penguin operators, ii) the CKM factors $\lambda_{p}^{(D)}=V_{p b} V_{p D}^{*}$ which contain the weak phase, and iii) the hadronic matrix elements $\langle f|\mathscr{O}| \bar{B}\rangle$ which include the strong phases. The interplay between the magnitudes and phases of these elements is at the heart of the rich and interesting phenomenology of non-leptonic charmless B decays. Together with the plethora of data for numerous observables such as branching ratios, direct $\mathrm{CP}$ asymmetries, and polarization fractions, this provides a fertile testing ground for the CKM mechanism and for QCD effects in hadronic decays.

The task of obtaining accurate theoretical predictions for non-leptonic B decays is complicated by the purely hadronic initial and final states, where QCD effects from many different scales arise. Several strategies have been developed to solve this problem, mostly based on flavour symmetries of the light quarks (see e.g. [1]) or on factorization, like PQCD [2,3] or QCD factorization (QCDF) [4-6]. The latter provides a rigorous and systematic framework to disentangle short- from long-distance physics in the heavy quark limit. The factorization formula reads

$$
\begin{aligned}
\left\langle M_{1} M_{2}\left|Q_{i}\right| \bar{B}\right\rangle & =\operatorname{im}_{B}^{2}\left\{F_{+}^{B \rightarrow M_{1}}(0) \int_{0}^{1} d u T_{i}^{I}(u) f_{M_{2}} \phi_{M_{2}}(u)+\left(M_{1} \leftrightarrow M_{2}\right)\right. \\
& \left.+\int_{0}^{\infty} d \omega \int_{0}^{1} d v d u T_{i}^{I I}(\omega, v, u) \hat{f}_{B} \phi_{B}(\omega) f_{M_{1}} \phi_{M_{1}}(v) f_{M_{2}} \phi_{M_{2}}(u)\right\} .
\end{aligned}
$$

The quantities $T_{i}^{I, I I}$ are the perturbatively calculable hard scattering kernels, where $T_{i}^{I}$ includes the so-called vertex corrections and starts at $\mathscr{O}(1)$, whereas $T_{i}^{I I}$ comprises the contributions from hard spectator scattering and starts at $\mathscr{O}\left(\alpha_{s}\right)$. The non-perturbative quantities are the $B \rightarrow M$ transition form factors $F_{+}^{B \rightarrow M}$, the decay constants $f_{j}$, and the distribution amplitudes $\phi_{k}$ of the heavy and light mesons. The QCDF formula (1.2) is valid to all orders in $\alpha_{s}$ and to leading power in $\Lambda_{\mathrm{QCD}} / m_{b}$. Moreover, the leading $\mathscr{O}\left(\alpha_{s}^{0}\right)$ term turns out to be real. Strong phases are thus either induced by perturbative contributions to the hard scattering kernels or by power-suppressed terms. Consequently, they are parametrically of order $\mathscr{O}\left(\alpha_{s}\left(m_{b}\right)\right)$ or $\mathscr{O}\left(\Lambda_{\mathrm{QCD}} / m_{b}\right)$.

The matrix elements in (1.2) are furthermore classified in terms of topological amplitudes, on which the various decay channels depend in different manners. For instance, one finds [7]

$$
\begin{aligned}
\sqrt{2}\left\langle\pi^{-} \pi^{0}\left|\mathscr{H}_{\text {eff }}\right| B^{-}\right\rangle & =A_{\pi \pi} \lambda_{u}^{(d)}\left[\alpha_{1}(\pi \pi)+\alpha_{2}(\pi \pi)\right], \\
-\left\langle\pi^{0} \pi^{0}\left|\mathscr{H}_{e f f}\right| \bar{B}^{0}\right\rangle & =A_{\pi \pi}\left\{\lambda_{u}^{(d)}\left[\alpha_{2}(\pi \pi)-\alpha_{4}^{u}(\pi \pi)\right]-\lambda_{c}^{(d)} \alpha_{4}^{c}(\pi \pi)\right\}, \\
\left\langle\pi^{-} \bar{K}^{0}\left|\mathscr{H}_{e f f}\right| B^{-}\right\rangle & =A_{\pi \bar{K}}\left[\lambda_{u}^{(s)} \alpha_{4}^{u}(\pi \bar{K})+\lambda_{c}^{(s)} \alpha_{4}^{c}(\pi \bar{K})\right] .
\end{aligned}
$$

Here, $\alpha_{1}$ and $\alpha_{2}$ are the colour-allowed and colour-suppressed tree amplitudes, respectively. $\alpha_{4}^{u, c}=$ $a_{4}^{u, c} \pm r_{\chi} a_{6}^{u, c}$ are the QCD penguin amplitudes, with $a_{4}^{u, c}$ as their leading-power part. The leading 
order (LO) and next-to-leading order (NLO) contributions to these amplitudes have been known since a long time $[4,6,7]$ and a comprehensive phenomenological analysis based on the NLO results was carried out in [7]. At the next-to-next-to-leading order (NNLO) the one-loop $\mathscr{O}\left(\alpha_{s}^{2}\right)$ correction to the hard spectator scattering [8-12], as well as the two-loop $\mathscr{O}\left(\alpha_{s}^{2}\right)$ correction to the tree topology of the vertex kernel [13-16] have been computed more than a decade ago. The first NNLO contribution to the vertex correction of the leading QCD penguin amplitudes that became available was the one-loop $\mathscr{O}\left(\alpha_{s}^{2}\right)$ insertion of the chromomagnetic dipole operator [17]. More recently the current-current operator contribution has been completed [18], and the remaining operator insertions are currently being finalized [19]. With these corrections at hand one can study the impact of QCD corrections to direct CP asymmetries.

\section{Theoretical framework}

The decays of heavy quarks are described in an effective five-flavour theory where the top quark and the heavy gauge bosons $W^{ \pm}, Z$ are integrated out. The resulting effective weak Hamiltonian for $b \rightarrow D$ transitions $(D=d, s)$ is given by [20,21]

$$
\mathscr{H}_{\text {eff }}=+\frac{4 G_{F}}{\sqrt{2}} \sum_{p=u, c} V_{p D}^{*} V_{p b}\left(C_{1} Q_{1}^{p}+C_{2} Q_{2}^{p}+\sum_{i=3}^{6} C_{i} Q_{i}+C_{8 g} Q_{8 g}\right)+\text { h.c. }
$$

The dimension-six operators in the so-called CMM basis [21] are defined as

$$
\begin{aligned}
Q_{1}^{p} & =\left(\bar{p}_{L} \gamma^{\mu} T^{A} b_{L}\right)\left(\bar{D}_{L} \gamma_{\mu} T^{A} p_{L}\right), & & Q_{5}=\left(\bar{D}_{L} \gamma^{\mu} \gamma^{v} \gamma^{\rho} b_{L}\right) \sum_{q}\left(\bar{q} \gamma_{\mu} \gamma_{v} \gamma_{\rho} q\right), \\
Q_{2}^{p} & =\left(\bar{p}_{L} \gamma^{\mu} b_{L}\right)\left(\bar{D}_{L} \gamma_{\mu} p_{L}\right), & Q_{6} & =\left(\bar{D}_{L} \gamma^{\mu} \gamma^{v} \gamma^{\rho} T^{A} b_{L}\right) \sum_{q}\left(\bar{q} \gamma_{\mu} \gamma_{v} \gamma_{\rho} T^{A} q\right), \\
Q_{3} & =\left(\bar{D}_{L} \gamma^{\mu} b_{L}\right) \sum_{q}\left(\bar{q} \gamma_{\mu} q\right), & & Q_{8 g}=\frac{-g_{s}}{32 \pi^{2}} m_{b} \bar{D} \sigma_{\mu v}\left(1+\gamma_{5}\right) G^{\mu v} b . \\
Q_{4} & =\left(\bar{D}_{L} \gamma^{\mu} T^{A} b_{L}\right) \sum_{q}\left(\bar{q} \gamma_{\mu} T^{A} q\right), & &
\end{aligned}
$$

In dimensional regularization the operator basis needs to be supplemented by a set of evanescent operators, for which we adopt the convention of $[22,23]$. The operators $Q_{1,2}^{p}$ are referred to as current-current operators, $Q_{3-6}$ are the QCD penguin operators, and $Q_{8 g}$ is the chromomagnetic dipole operator.

The hard scattering kernels will be extracted via a matching procedure from QCD onto softcollinear effective theory (SCET). We denote the collinear and anti-collinear SCET fields by $\chi$ and $\xi$, respectively. The only physical SCET operator has the fermion contraction $(\bar{\chi} \chi)\left(\bar{\xi} h_{v}\right)$ and is given by

$$
O_{1}=\left(\bar{\chi} \frac{h_{-}}{2}\left(1-\gamma_{5}\right) \chi\right)\left(\bar{\xi}_{\not h_{+}}\left(1-\gamma_{5}\right) h_{v}\right)
$$

where $h_{v}$ denotes the HQET heavy-quark field. In contrast, the diagrams relevant to the penguin amplitudes lead to operators where the fermion lines are contracted in the Fierz ordering $(\bar{\xi} \chi)\left(\bar{\chi} h_{v}\right)$. The corresponding SCET operators are chosen as

$$
\tilde{O}_{n}=\left(\bar{\xi} \gamma_{\perp}^{\alpha} \gamma_{\perp}^{\mu_{1}} \gamma_{\perp}^{\mu_{2}} \ldots \gamma_{\perp}^{\mu_{2 n-2}} \chi\right)\left(\bar{\chi}\left(1+\gamma_{5}\right) \gamma_{\perp \alpha} \gamma_{\perp \mu_{2 n-2}} \gamma_{\perp \mu_{2 n-3}} \ldots \gamma_{\perp \mu_{1}} h_{v}\right)
$$


They are evanescent for $n>1$, and $\tilde{O}_{1}$ is Fierz-equivalent to $O_{1} / 2$ in four dimensions. We therefore add $\tilde{O}_{1}-O_{1} / 2$ as another evanescent SCET operator.

By imposing the matching condition one can derive master formulas for the extraction of the hard scattering kernels $\widetilde{T}_{i}^{(\ell)}$ for each operator $Q_{i}$. At tree level, one and two loops they read, respectively,

$$
\begin{aligned}
\widetilde{T}_{i}^{(0)}= & \widetilde{A}_{i 1}^{(0)} \\
\widetilde{T}_{i}^{(1)}= & \widetilde{A}_{i 1}^{(1) \mathrm{nf}}+Z_{i j}^{(1)} \widetilde{A}_{j 1}^{(0)}+\underbrace{\widetilde{A}_{i 1}^{(1) \mathrm{f}}-A_{31}^{(1) \mathrm{f}} \widetilde{A}_{i 1}^{(0)}}_{\mathscr{O}(\varepsilon)}-\underbrace{\left[\widetilde{Y}_{11}^{(1)}-Y_{11}^{(1)}\right] \widetilde{A}_{i 1}^{(0)}}_{\mathscr{O}(\varepsilon)}-\underbrace{\sum_{b>1} \widetilde{A}_{i b}^{(0)} \widetilde{Y}_{b 1}^{(1)}}_{\mathscr{O}(\varepsilon)}, \\
\widetilde{T}_{i}^{(2)}= & \widetilde{A}_{i 1}^{(2) \mathrm{nf}}+Z_{i j}^{(1)} \widetilde{A}_{j 1}^{(1)}+Z_{i j}^{(2)} \widetilde{A}_{j 1}^{(0)}+Z_{\alpha}^{(1)} \widetilde{A}_{i 1}^{(1) \mathrm{nf}} \\
& +(-i) \delta m^{(1)} \widetilde{A}_{i 1}^{(1) \mathrm{nf}}+Z_{e x t}^{(1)}\left[\widetilde{A}_{i 1}^{(1) \mathrm{nf}}+Z_{i j}^{(1)} \widetilde{A}_{j 1}^{(0)}\right] \\
& -\widetilde{T}_{i}^{(1)}\left[C_{F F}^{(1)}+\widetilde{Y}_{11}^{(1)}\right]-\sum_{b>1} \widetilde{H}_{i b}^{(1)} \widetilde{Y}_{b 1}^{(1)} \\
& +\left[\widetilde{A}_{i 1}^{(2) \mathrm{f}}-A_{31}^{(2) \mathrm{f}} \widetilde{A}_{i 1}^{(0)}\right]+(-i) \delta m^{(1)}\left[\widetilde{A}_{i 1}^{(1) \mathrm{f}}-A_{31}^{(1) \mathrm{f}} \widetilde{A}_{i 1}^{(0)}\right] \\
& +\left(Z_{\alpha}^{(1)}+Z_{e x t}^{(1)}\right)\left[\widetilde{A}_{i 1}^{(1) \mathrm{f}}-A_{31}^{(1) \mathrm{f}} \widetilde{A}_{i 1}^{(0)}\right] \\
& -\left[\widetilde{M}_{11}^{(2)}-M_{11}^{(2)}\right] \widetilde{A}_{i 1}^{(0)} \\
& -\left(C_{F F}^{(1)}-\xi_{45}^{(1)}\right)\left[\widetilde{Y}_{11}^{(1)}-Y_{11}^{(1)}\right] \widetilde{A}_{i 1}^{(0)}-\left[\widetilde{Y}_{11}^{(2)}-Y_{11}^{(2)}\right] \widetilde{A}_{i 1}^{(0)} \\
& -\sum_{b>1} \widetilde{A}_{i b}^{(0)} \widetilde{M}_{b 1}^{(2)}-\sum_{b>1} \widetilde{A}_{i b}^{(0)} \widetilde{Y}_{b 1}^{(2)} .
\end{aligned}
$$

The detailed derivation of the master formulas and a comprehensive explanation of the notation can be found in $[15,19]$. Here we give merely the meaning of the most important quantities. The $\widetilde{A}_{i a}^{(\ell)}$ denote bare $\ell$-loop on-shell matrix elements of QCD operators (index $i$ ), whose result is proportional to $\tilde{O}_{a}$ on the SCET side. For the matching procedure it turns out to be convenient to further split up the amplitudes $\widetilde{A}_{i a}^{(\ell)}$ into factorizable (superscript f) and non-factorizable (superscript nf) diagrams, see [15] for details. The renormalization factors $Z_{i j}, Z_{\alpha}, \delta m$, and $Z_{\text {ext }}$ account for operator, coupling, mass, and wave-function renormalization, respectively. In the matrices $Z_{i j}$ the column index $j$ runs over both, physical and evanescent operators. $C_{F F}^{(1)}$ [15] can be determined from one-loop matching calculations for heavy-to-light transitions, and the quantity $\xi_{45}^{(1)}$ [24] gives the relation between the four- and five-flavour coupling in $D$ dimensions. The $\widetilde{Y}_{a b}^{(\ell)}$ are renormalization factors in SCET which also take the mixing of physical into evanescent SCET operators into account.

\section{The two-loop calculation}

The calculation of the NNLO correction to the vertex-kernel of the leading QCD penguin amplitudes $a_{4}^{u}$ and $a_{4}^{c}$ amounts to the evaluation of $\sim 130$ Feynman diagrams. A subset of them 

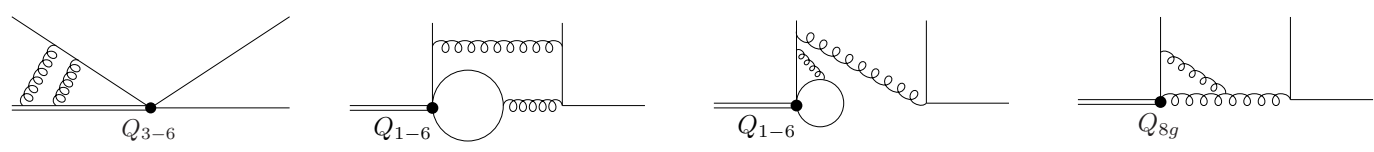

Figure 1: Sample diagrams at NNLO. The diagram on the right denotes a one-loop $\mathscr{O}\left(\alpha_{s}^{2}\right)$ contribution from the chromomagnetic dipole operator $Q_{8 g}[17]$.

is shown in Fig. 1. The one-loop $\mathscr{O}\left(\alpha_{s}^{2}\right)$ contribution of the chromomagnetic dipole operator $Q_{8 g}$, depicted in the right panel in Fig. 1, was calculated in [17]. All other contributions are genuine two-loop diagrams. Moreover, the operators from the effective weak Hamiltonian contribute with several insertions to the penguin amplitudes. They are depicted in Fig. 2 and lead to quite some bookkeeping during the calculation.

The kinematics of the process can be inferred from Fig. 3. The external states satisfy $p_{b}^{2}=m_{b}^{2}$ and $p^{2}=q^{2}=0$, and the fermion in the loop (solid circle) can have mass $m_{f}=0$ (light quarks), $m_{f}=m_{c}$ or $m_{f}=m_{b}$. The problem therefore depends on two dimensionless variables, the momentum fraction $\bar{u}=1-u \in[0,1]$, and the mass ratio $z_{f} \equiv m_{f}^{2} / m_{b}^{2}$. There is a kinematic threshold at $\bar{u}=4 z_{f}$. For later convenience we also introduce the variables $r=\sqrt{1-4 z_{c}}$ and $s=\sqrt{1-4 z_{c} / \bar{u}}$. The reduction of the amplitude is done by techniques that have become standard in multi-loop computations. We work in dimensional regularization with $D=4-2 \varepsilon$, reduce the tensor structure via Passarino-Veltman relations, followed by IBP reduction [25-27] of the scalar integrals to master integrals using FIRE [28] and an in-house routine. This procedure results in about three dozens of yet unknown master integrals.

The master integrals that stem from the insertion of penguin operators into the "tree-type" diagrams (left panel of Fig 1) were computed more than a decade ago [13-15, 29] and evaluate to harmonic polylogarithms (HPLs). The remaining ones were computed analytically in [30] in a
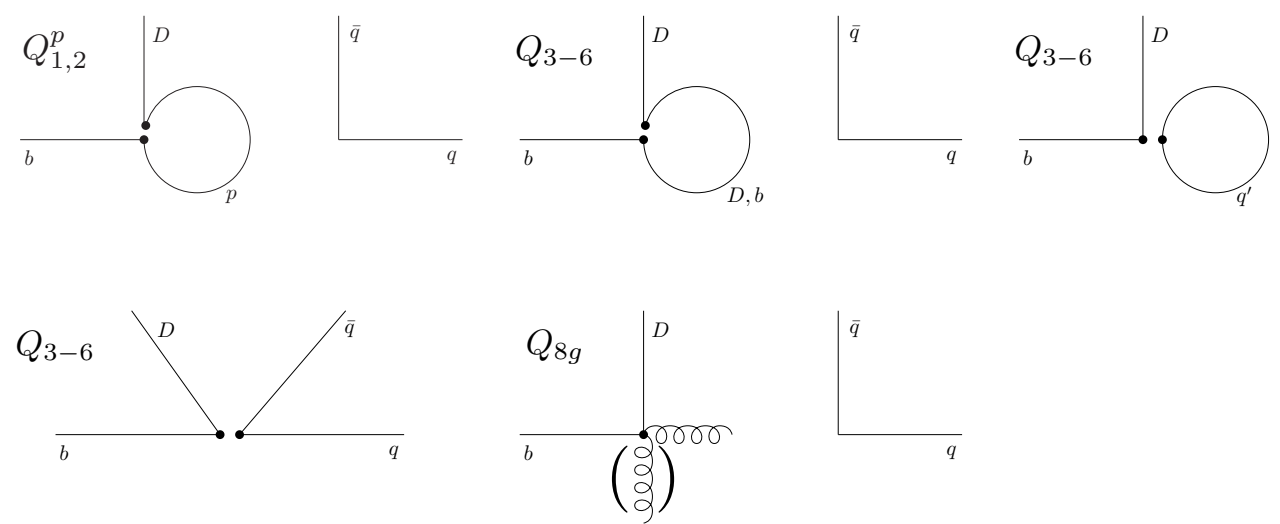

Figure 2: Graphical illustration of the various operator insertions. The symbols stand for $p \in\{u, c\}$, $D \in\{d, s\}, q \in\{u, d, s\}$ and $q^{\prime} \in\{u, d, s, c, b\}$. The black dots denote the operator insertion from the effective weak Hamiltonian. Disconnected parts are understood to be connected by gluons. 


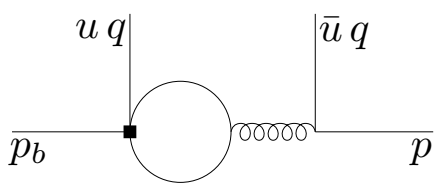

Figure 3: Kinematics of charmless non-leptonic two-body decays in QCD factorization.

canonical basis [31] in terms of iterated integrals (Goncharov polylogarithms) over the alphabet

$$
\left\{0, \pm 1, \pm 3, \pm i \sqrt{3}, \pm r, \pm \frac{r^{2}+1}{2}, \pm\left(1+2 \sqrt{z_{c}}\right), \pm\left(1-2 \sqrt{z_{c}}\right)\right\} .
$$

Most of the individual terms in the master formulas (2.5) - (2.7) have poles in $\varepsilon$, which have to cancel in the total expression for the hard scattering kernels $\widetilde{T}_{i}^{(\ell)}$. We checked the pole cancellation analytically through to $\mathscr{O}\left(\alpha_{s}^{2}\right)$ and for all operators $i \in\left\{Q_{1}^{u}, Q_{2}^{u}, Q_{1}^{c}, Q_{2}^{c}, Q_{3-6}, Q_{8 g}\right\}$. Also the finite parts of the hard scattering kernels were obtained completely analytically.

Having the hard scattering kernels completely analytically catalyses the convolution with the light-meson light-cone distribution amplitude $\phi_{M}(u)$ considerably. The latter is expanded according to

$$
\phi_{M}(u)=6 u \bar{u}\left[1+\sum_{n=1}^{\infty} a_{n}^{M} C_{n}^{(3 / 2)}(2 u-1)\right]
$$

where $a_{n}^{M} \equiv a_{n}^{M}(\mu)$ and $C_{n}^{(3 / 2)}(x)$ are the Gegenbauer moments and polynomials, respectively. Truncating the Gegenbauer expansion at $n=2$ is sufficient in practice.

The convolution of those parts of the hard scattering kernels that come from "tree-type" diagrams is known from the NNLO calculation of the tree amplitudes [13-15] and can be done completely analytically. For the "penguin-type" diagrams the majority of the terms can be convoluted analytically by substituting $s=\sqrt{1-4 z_{c} / \bar{u}}$,

$$
\int_{0}^{1} d u \widetilde{T}_{i}(u) \phi_{M}(u)=\int_{r}^{+i \infty} d s \frac{2 s\left(r^{2}-1\right)}{\left(1-s^{2}\right)^{2}} \widetilde{T}_{i}(u(s)) \phi_{M}(u(s)),
$$

because it leads to iterated integrals over the same alphabet (3.1). The threshold at $\bar{u}=4 z_{c}$ is mapped to $s=0$. Subtleties arise when taking the limits $s \rightarrow r$ and $s \rightarrow+i \infty$. In case of the lower limit individual terms contain power divergences proportional to $1 /(r-s)^{n}$ which can be isolated via a Taylor expansion about $s=r$ of the corresponding numerators and disappear in the sum of all terms. In the case of the upper limit logarithmic divergences contained in Goncharov polylogarithms have to be isolated, which is done via an argument inversion with $z=i$ as pivot point. The divergences that arise in individual terms as $s \rightarrow+i \infty$ have to cancel in the end as well. This procedure yields Goncharov polylogarithms up to weight five, which are evaluated numerically with GiNaC [32,33].

In this way, we obtain all $\mu$-dependent terms in the penguin amplitudes completely analytically. The $\mu$-independent pieces are obtained as accurate interpolations in $z_{c}$. 


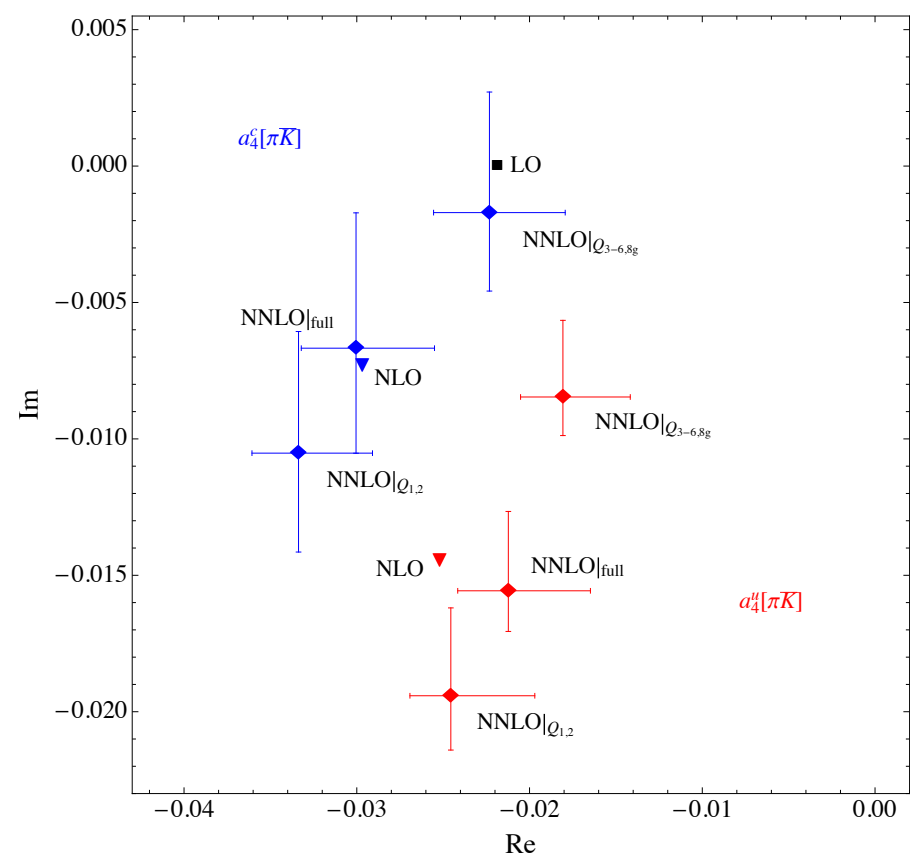

Figure 4: Anatomy of QCD corrections to $a_{4}^{u}$ and $a_{4}^{c}$. See text for details on the data points.

\section{Preliminary results and conclusion}

Since the publication of the results presented here is still pending, all results shown are preliminary. The numerical values for the leading QCD penguin amplitudes $a_{4}^{u}$ and $a_{4}^{c}$ are obtained to NNLO by using the same input parameters as in [18], and for the $\pi \bar{K}$ final state result in

$$
\begin{aligned}
a_{4}^{u}(\pi \bar{K}) / 10^{-2}= & -2.87-[0.09+0.09 i]_{\mathrm{V}_{1}}+[0.49-1.32 i]_{\mathrm{P}_{1}} \\
& -[0.32+0.71 i]_{\mathrm{P}_{2}, \mathrm{Q}_{1,2}^{u}}+[0.33+0.38 i]_{\mathrm{P}_{2}, \mathrm{Q}_{3-6,8 g}} \\
& +\left[\frac{r_{\mathrm{sp}}}{0.434}\right]\left\{[0.13]_{\mathrm{LO}}+[0.14+0.12 i]_{\mathrm{HV}}-[0.01-0.05 i]_{\mathrm{HP}}+[0.07]_{\mathrm{tw} 3}\right\} \\
= & \left(-2.12_{-0.29}^{+0.48}\right)+\left(-1.56_{-0.15}^{+0.29}\right) i, \\
a_{4}^{c}(\pi \bar{K}) / 10^{-2}= & -2.87-[0.09+0.09 i]_{\mathrm{V}_{1}}+[0.05-0.62 i]_{\mathrm{P}_{1}} \\
& -[0.77+0.50 i]_{\mathrm{P}_{2}, \mathrm{Q}_{1,2}^{\mathrm{c}}}+[0.33+0.38 i]_{\mathrm{P}_{2}, \mathrm{Q}_{3-6,8 g}} \\
& +\left[\frac{r_{\mathrm{sp}}}{0.434}\right]\left\{[0.13]_{\mathrm{LO}}+[0.14+0.12 i]_{\mathrm{HV}}+[0.01+0.03 i]_{\mathrm{HP}}+[0.07]_{\mathrm{tw} 3}\right\} \\
= & \left(-3.00_{-0.32}^{+0.45}\right)+\left(-0.67_{-0.39}^{+0.50}\right) i .
\end{aligned}
$$

Both amplitudes receive identical contributions at leading order (LO), which is real. The second and third term in the first line make up for the NLO result. The second line of each equation is 

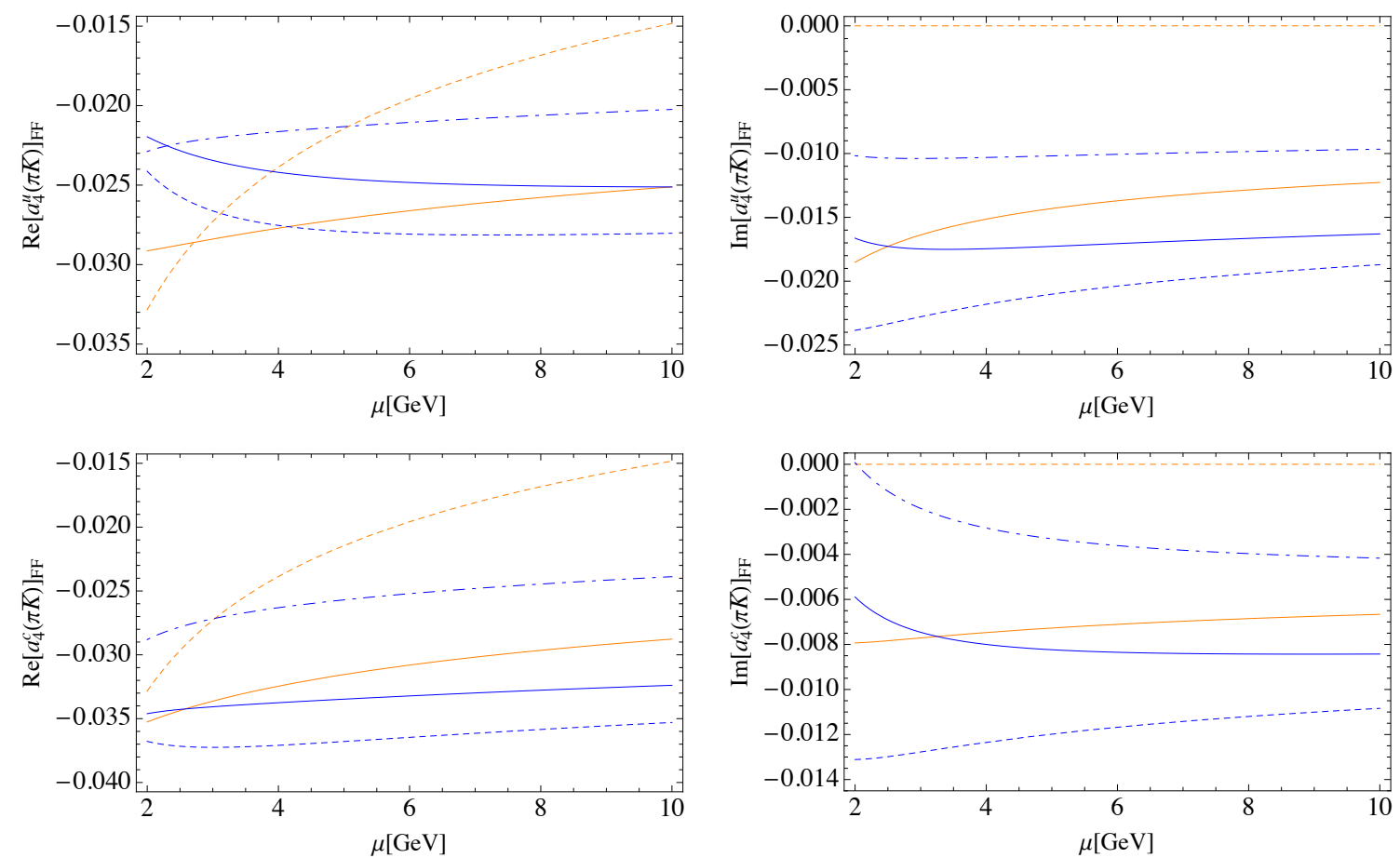

Figure 5: Scale dependence of $a_{4}^{u}$ (upper two panels) and $a_{4}^{c}$ (lower two panels). The curves show only the form factor (FF) contribution without spectator scattering. Orange lines represent results to LO (dashed) and NLO (solid), respectively. The dashed (dash-dotted) blue lines denote NNLO results with only currentcurrent (only penguin and chromomagnetic dipole) operators included, while the blue solid lines show the full NNLO result.

the NNLO result, which is split up into the contibution from the current-current operators $Q_{1,2}^{p}$ [18] and the penguin plus chromomagnetic dipole operators $Q_{3-6,8 g}$ [19]. All terms that multiply

$$
r_{\mathrm{sp}}=\frac{9 f_{\pi} \hat{f}_{B}}{m_{b} \lambda_{B} F_{+}^{B \rightarrow \pi}(0)}
$$

arise from hard spectator scattering and are sensitive to the first inverse moment $\lambda_{B}$ of the B meson distribution amplitude. The total error in the last line contains parametric and perturbative uncertainties added in quadrature [18]. From the NNLO numbers one can clearly see that the contribution from the penguin operators and $Q_{8 g}$ tends to cancel that of the current-current operators and therefore the NNLO result is close to the NLO one. The situation is depicted graphically in Fig. 4 where, however, the LO and NLO data points refer to the result obtained in the BBL operator basis [20] and hence cannot be trivially reproduced using the numbers in (4.1) and (4.2) which were computed in the CMM basis [21] . The leading order point "LO" (black square) is common to both $a_{4}^{u}$ and $a_{4}^{c}$, whereas the triangles labelled "NLO" show the respective results through to NLO. The data points for $a_{4}^{u}$ and $a_{4}^{c}$ are indicated in red and blue, respectively. The diamonds with error bars labelled "NNLO" show the results through to NNLO with only $Q_{1,2}$, only $Q_{3-6,8 g}$, and all contributions included, respectively.

Fig. 5 shows the scale dependence of the vertex correction of $a_{4}^{u}$ (upper two panels) and $a_{4}^{c}$ (lower two panels). Note that, like in Fig. 4, the LO and NLO curves refer to the result obtained 
in the BBL basis. One can clearly see a reduction of the scale dependence from the LO (orange dashed) to the NLO (orange solid) and finally the NNLO (blue solid) curves. The reduction is slightly less pronounced in the imaginary parts since NNLO is in fact only their first correction.

To conclude, we completed the calculation of the leading penguin amplitudes in QCD factorization to NNLO. The computation is done via a matching from QCD onto SCET and is complicated by the presence of evanescent operators in both theories. During the calculation one has to deal with a genuine two-loop, two-scale problem which we solved almost completely analytically. The NNLO correction turns out to be numerically small, with a cancellation between the contributions from current-current and penguin operators. The precise impact of the NNLO correction on the phenomenology of charmless two-body non-leptonic B decays will be investigated in the future.

\section{Acknowledgments}

We would like to thank the organisers of "RADCOR 2019" for creating a very pleasant and inspiring atmosphere. The work of GB and TH was supported by DFG Forschergruppe FOR 1873 "Quark Flavour Physics and Effective Field Theories". The work of MB is supported by the DFG Sonderforschungsbereich/Transregio 110 "Symmetries and the Emergence of Structure in QCD". The work of XL is supported by the National Natural Science Foundation of China under Grant Nos. 11675061 and 11435003, and by the Fundamental Research Funds for the Central Universities under Grant No. CCNU18TS029.

\section{References}

[1] D. Zeppenfeld, SU(3) Relations for B Meson Decays, Z. Phys. C8 (1981) 77.

[2] Y.-Y. Keum, H.-n. Li and A. I. Sanda, Fat penguins and imaginary penguins in perturbative QCD, Phys. Lett. B504 (2001) 6-14, [hep-ph/ 0004004 ].

[3] C.-D. Lü, K. Ukai and M.-Z. Yang, Branching ratio and $C P$ violation of $B \longrightarrow$ pi pi decays in perturbative QCD approach, Phys. Rev. D63 (2001) 074009, [hep-ph/ 0004213 ].

[4] M. Beneke, G. Buchalla, M. Neubert and C. T. Sachrajda, $Q C D$ factorization for $B \rightarrow \pi \pi$ decays: Strong phases and CP violation in the heavy quark limit, Phys. Rev. Lett. 83 (1999) 1914-1917, [hep-ph/9905312].

[5] M. Beneke, G. Buchalla, M. Neubert and C. T. Sachrajda, QCD factorization for exclusive, nonleptonic B meson decays: General arguments and the case of heavy light final states, Nucl. Phys. B591 (2000) 313-418, [hep-ph/ 0006124$].$

[6] M. Beneke, G. Buchalla, M. Neubert and C. T. Sachrajda, $Q C D$ factorization in $B \rightarrow \pi K, \pi \pi$ decays and extraction of Wolfenstein parameters, Nucl. Phys. B606 (2001) 245-321, [hep-ph/ 0104110$].$

[7] M. Beneke and M. Neubert, $Q C D$ factorization for $B \rightarrow P P$ and $B \rightarrow P V$ decays, Nucl. Phys. B675 (2003) 333-415, [hep-ph/ 0308039 ].

[8] M. Beneke and S. Jäger, Spectator scattering at NLO in non-leptonic b decays: Tree amplitudes, Nucl. Phys. B751 (2006) 160-185, [hep-ph / 0512351].

[9] M. Beneke and S. Jäger, Spectator scattering at NLO in non-leptonic B decays: Leading penguin amplitudes, Nucl. Phys. B768 (2007) 51-84, [hep-ph / 0610322$].$ 
[10] N. Kivel, Radiative corrections to hard spectator scattering in B $\rightarrow \pi \pi$ decays, JHEP 05 (2007) 019, [hep-ph/0608291].

[11] V. Pilipp, Hard spectator interactions in $B \rightarrow \pi \pi$ at $\mathscr{O}\left(\alpha_{s}^{2}\right)$, Nucl. Phys. B794 (2008) 154-188, [0709.3214].

[12] A. Jain, I. Z. Rothstein and I. W. Stewart, Penguin Loops for Nonleptonic B-Decays in the Standard Model: Is there a Penguin Puzzle?, 0706.3399.

[13] G. Bell, NNLO vertex corrections in charmless hadronic B decays: Imaginary part, Nucl. Phys. B795 (2008) 1-26, [0705.3127].

[14] G. Bell, NNLO vertex corrections in charmless hadronic B decays: Real part, Nucl. Phys. B822 (2009) 172-200, [0 902.1915$].$

[15] M. Beneke, T. Huber and X.-Q. Li, NNLO vertex corrections to non-leptonic B decays: Tree amplitudes, Nucl. Phys. B832 (2010) 109-151, [0911.3655].

[16] G. Bell and V. Pilipp, $B^{-} \rightarrow \pi^{-} \pi^{0} / \rho^{-} \rho^{0}$ to NNLO in QCD factorization, Phys. Rev. D80 (2009) 054024, [0907.1016].

[17] C. S. Kim and Y. W. Yoon, Order $\alpha_{s}^{2}$ magnetic penguin correction for B decay to light mesons, JHEP 11 (2011) 003, [1107.1601].

[18] G. Bell, M. Beneke, T. Huber and X.-Q. Li, Two-loop current-current operator contribution to the non-leptonic QCD penguin amplitude, Phys. Lett. B750 (2015) 348-355, [1507.03700].

[19] G. Bell, M. Beneke, T. Huber and X.-Q. Li, in preparation, .

[20] G. Buchalla, A. J. Buras and M. E. Lautenbacher, Weak decays beyond leading logarithms, Rev. Mod. Phys. 68 (1996) 1125-1144, [hep-ph/9512380].

[21] K. G. Chetyrkin, M. Misiak and M. Münz, $|\Delta F|=1$ nonleptonic effective Hamiltonian in a simpler scheme, Nucl. Phys. B520 (1998) 279-297, [hep-ph/9711280].

[22] P. Gambino, M. Gorbahn and U. Haisch, Anomalous dimension matrix for radiative and rare semileptonic B decays up to three loops, Nucl. Phys. B673 (2003) 238-262, [hep-ph/ 0306079 ].

[23] M. Gorbahn and U. Haisch, Effective Hamiltonian for non-leptonic $|\Delta F|=1$ decays at NNLO in QCD, Nucl. Phys. B713 (2005) 291-332, [hep-ph / 0411071$].$

[24] M. Beneke, T. Huber and X. Q. Li, Two-loop QCD correction to differential semi-leptonic $b \rightarrow u$ decays in the shape-function region, Nucl. Phys. B811 (2009) 77-97, [0810 . 1230].

[25] F. V. Tkachov, A Theorem on Analytical Calculability of Four Loop Renormalization Group Functions, Phys. Lett. 100B (1981) 65-68.

[26] K. G. Chetyrkin and F. V. Tkachov, Integration by Parts: The Algorithm to Calculate beta Functions in 4 Loops, Nucl. Phys. B192 (1981) 159-204.

[27] S. Laporta, High precision calculation of multiloop Feynman integrals by difference equations, Int. J. Mod. Phys. A15 (2000) 5087-5159, [hep-ph/ 0102033$].$

[28] A. V. Smirnov, Algorithm FIRE - Feynman Integral REduction, JHEP 10 (2008) 107, [0 807.3243 ].

[29] T. Huber, On a two-loop crossed six-line master integral with two massive lines, JHEP 03 (2009) 024, [0901.2133].

[30] G. Bell and T. Huber, Master integrals for the two-loop penguin contribution in non-leptonic B-decays, JHEP 12 (2014) 129, [1410.2804]. 
[31] J. M. Henn, Multiloop integrals in dimensional regularization made simple, Phys. Rev. Lett. 110 (2013) 251601, [1304.1806].

[32] C. W. Bauer, A. Frink and R. Kreckel, Introduction to the GiNaC framework for symbolic computation within the C++ programming language, J. Symb. Comput. 33 (2000) 1, [cs/ 0004015 ].

[33] J. Vollinga and S. Weinzierl, Numerical evaluation of multiple polylogarithms, Comput. Phys. Commun. 167 (2005) 177, [hep-ph/ 041025 9]. 